\title{
Nanoadhesion of elastic bodies: Roughness and temperature effects
}

\author{
S. Zilberman \\ School of Chemistry, Tel Aviv University, Tel Aviv, Israel 69978 and IFF, FZ-Jülich, \\ 52425 Jülich, Germany \\ B. N. J. Persson a) \\ IFF, FZ-Jülich, 52425 Jülich, Germany
}

(Received 19 August 2002; accepted 14 January 2003)

\begin{abstract}
We present a simple model which illustrates the nature of the contact between an elastic solid and a hard surface with cosine-corrugation profile. In the continuum limit, the contact mechanics depends only on two dimensionless parameters, namely the ratio between the height and wavelength of the substrate corrugation, and the ratio between a surface energy and an elastic energy. The theory shows that the complete contact state is always a local energy minima (in the zero temperature limit), but for large enough surface roughness the global minima correspond to a partial contact state. We show that at nonzero temperature, the contribution to the free energy from the vibrational entropy is very important, and favors the detached state. Computer simulations results are also presented where we study more complicated roughness geometries and the influence of temperature on the adhesion. Simulation results agrees well with the analytical predictions. (C) 2003 American Institute of Physics. [DOI: 10.1063/1.1558038]
\end{abstract}

\section{INTRODUCTION}

Even a highly polished surface has surface roughness on many different length scales. When two bodies with nominally flat surfaces are brought into contact, the area of real contact will usually only be a small fraction of the nominal contact area. We can visualize the contact regions as small areas where asperities from one solid are squeezed against asperities of the other solid. The area of real contact depends not only on the pressure by which the solids are squeezed together, but also on the adhesion interaction between the surfaces. ${ }^{1-4}$

One of us (B.N.J.P.) has developed a theory of contact mechanics, ${ }^{5}$ valid for randomly rough surfaces, but neglecting adhesion. We have also studied the role of adhesion, both when the contact between the two solids is complete, ${ }^{6}$ and when partial contact occur on many length scales. ${ }^{7}$ Adhesion is particularly important for elastically soft solids, e.g., rubber or gelatin, where it may pull the two solids in direct contact over the whole nominal contact area.

In this paper we consider the adhesion between an elastically compliant solid (referred to as block) with a flat surface and a rigid substrate with a cosine-corrugation profile. In a previous publication ${ }^{8}$ we have presented some results for this model in the limit where thermal effects can be ignored. Here we consider finite temperatures, and show that the vibrational entropy gives a very important contribution to the free energy, which favors the detached state. These thermal effects are studied both analytically and via computer simulations.

The influence of surface roughness on the adhesion between rubber (or any other elastic solid) and a hard substrate has been studied in a classic paper by Fuller and Tabor. ${ }^{9}$ They found that already a relative small surface roughness

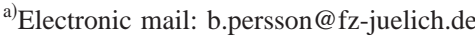

can completely remove the adhesion. In order to understand the experimental data they developed a very simple model where the adhesion force was obtained by applying the contact theory of Johnson, Kendall, and Roberts ${ }^{10}$ to each individual asperity. We note that the formalism used by Fuller and Tabor is only valid at "high" surface roughness, where the area of real contact (and the adhesion force) is very small. The theory presented in the following is accurate for any amplitude of the surface roughness, thus accounting also for "small" roughness, where the area of real contact is of the same order of magnitude as the nominal contact area.

Johnson ${ }^{12}$ and Hui and co-workers, ${ }^{11}$ have studied a similar model as considered in our paper. These authors calculated the pull-off force and the contact area between a semi-infinite elastic (or viscoelastic) solid with a wavy surface, and a semi-infinite hard flat substrate.

We instead focus on the influence of surface roughness on the adhesion between two solids of in principle arbitrary shape. We assume that the wavelength of the surface roughness profile is small compared with the diameter of the nominal contact area. Under these conditions the pull-off force is given by the standard formulas for the adhesion between solid objects of different shapes (e.g., spheres or cubic blocks, see Ref. 2) but with an interfacial free energy $\gamma_{\text {eff }}$ which depends on the surface roughness as outlined in the following. This is a fundamentally different problem from the one studied in Refs. 12 and 11, and we believe that our results are the first exact results in this context. Moreover, we take into account the thermal vibrations of the surfaces in contact, thus enabling us to study the finite temperature entropic contributions to the effective surface energy.

We present nanoscale molecular dynamics simulation, and compare them to the continuum mechanics treatment. The simulations demonstrate the importance of proper treatment of thermal effects in contact mechanics. 


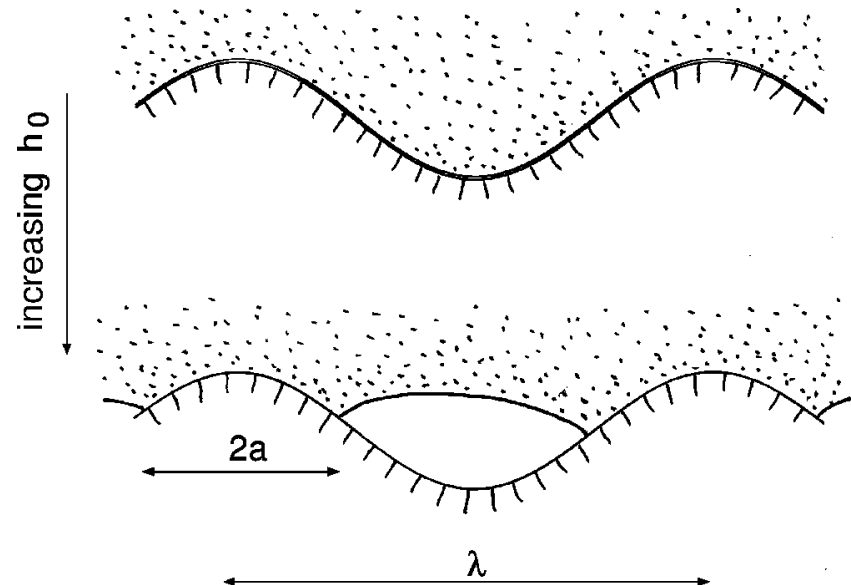

FIG. 1. The detachment transition (schematic). For small surface roughness, complete contact occurs in the nominal contact area (top), while for large surface roughness there is a jump to partial contact (bottom).

\section{CONTACT MECHANICS THEORY ( $T=0 \mathrm{~K})$}

We present first a qualitative discussion about the role of the block-substrate adhesion interaction. When the block deforms and fills out a surface cavity of width $\lambda$ and depth $h$ $\ll \lambda$ of the substrate, an elastic energy $U_{\mathrm{el}} \approx E \lambda h^{2}$ (where $E$ is the elastic modulus) will be stored in the block. Now, if this elastic energy is smaller than the gain in adhesion energy $U_{\mathrm{ad}} \approx-\Delta \gamma \lambda^{2}$, where $-\Delta \gamma$ is the change of surface free energy per unit area upon contact due to the block-substrate interaction, then (even in the absence of the load) the block will deform spontaneously to fill out the substrate cavities. A very important parameter is thus the ratio $\Theta=-U_{\mathrm{el}} / U_{\mathrm{ad}}$ $\approx E h^{2} / \Delta \gamma \lambda$. If $\Theta \ll 1$ the block will fill out the roughness cavities resulting in complete contact, while for $\Theta \gg 1$ the contact (in the absence of an external load) will only occur at the top of the highest surface asperities.

Let us consider a block with a smooth flat surface in contact with a hard substrate with a cosine-corrugation, $z$ $=h_{0} \cos (2 \pi x / \lambda)$, in the $x$ direction and constant in the $y$ direction. For small amplitude of the corrugation we expect complete contact between the solids at the interface, while only partial contact occurs for large enough corrugation, see Fig. 1. If the block is in contact with the substrate along strips of width $2 a$ at the top of the cosine profile (see Fig. 1), then the local pressure distribution equals ${ }^{13,14}$

$$
p(x)=p_{1}(x)+p_{2}(x),
$$

where

$$
\begin{aligned}
& p_{1}=2 \pi E^{*} \frac{h_{0}}{\lambda} \cos \left(\frac{\pi x}{\lambda}\right)\left[\sin ^{2}\left(\frac{\pi a}{\lambda}\right)-\sin ^{2}\left(\frac{\pi x}{\lambda}\right)\right]^{1 / 2}, \\
& p_{2}=-\sigma_{0}\left[1-\left(\frac{\cos (\pi a / \lambda)}{\cos (\pi x / \lambda)}\right)^{2}\right]^{-1 / 2}
\end{aligned}
$$

for $-a<x<a$ and zero otherwise. Here $p_{1}(x)$ is the pressure distribution which gives rise to a cosine deformation in the contact region $-a<x<a$, while $p_{2}(x)$ gives rise to a constant deformation in the contact region. We have defined $E^{*}=E /\left(1-\nu^{2}\right)$ where $\nu$ is the Poisson ratio. The average pressure

$$
\begin{aligned}
& \bar{p}=\frac{1}{\lambda} \int_{-a}^{a} p(x)=\bar{p}_{1}+\bar{p}_{2}, \\
& \bar{p}_{1}=\pi E^{*} \frac{h_{0}}{\lambda} \sin ^{2}\left(\frac{\pi a}{\lambda}\right), \quad \bar{p}_{2}=-\sigma_{0} .
\end{aligned}
$$

The pressure $\sigma_{0}$ is determined so that $\bar{p}=\sigma$, where $\sigma$ is the external applied stress. This gives

$$
\sigma_{0}=\pi E^{*} \frac{h_{0}}{\lambda} \sin ^{2}\left(\frac{\pi a}{\lambda}\right)-\sigma .
$$

In what follows we focus on the case $\sigma=0$.

The elastic displacement of the block surface

$$
u_{z}=u_{z}^{(1)}+u_{z}^{(2)} .
$$

The displacement $u_{z}^{(1)}$ induced by $p_{1}$ is given by ${ }^{13}$

$$
u_{z}^{(1)}=h_{0} \cos \left(\frac{2 \pi x}{\lambda}\right)+2 h_{0} \sin ^{2}\left(\frac{\pi a}{\lambda}\right) F(x),
$$

where $F=0$ for $|x|<a$ while for $a<|x|<\lambda / 2$,

$$
F(x)=\xi\left(\xi^{2}-1\right)^{1 / 2}-\ln \left[\xi+\left(\xi^{2}-1\right)^{1 / 2}\right],
$$

with

$$
\xi(x)=\frac{\sin (\pi x / \lambda)}{\sin (\pi a / \lambda)} .
$$

The displacement $u_{z}^{(2)}$ induced by $p_{2}$ is given by ${ }^{14}$

$$
u_{z}^{(2)}=\frac{2}{\pi} \lambda \frac{\sigma_{0}}{E^{*}} \ln \left[\xi+\left(\xi^{2}-1\right)^{1 / 2}\right]
$$

for $a<|x| \leqslant \lambda / 2$ and $u_{z}^{(2)}=0$ for $|x|<a$.

The elastic energy induced by the surface roughness and stored at the interface equals

$$
U_{\mathrm{el}}=\frac{1}{2} \int d^{2} x[p(x)-\bar{p}] u_{z}(x),
$$

and the adhesion energy is given by

$$
U_{\mathrm{ad}}=-A_{0} \Delta \gamma \frac{1}{\lambda} \int_{-a}^{a} d x\left[1+\left(\frac{d u_{z}(x)}{d x}\right)^{2}\right]^{1 / 2},
$$

with $A_{0}$ being the nominal contact area. Using the abovementioned equations gives the total energy

$$
\begin{aligned}
U=U_{\mathrm{el}}+U_{\mathrm{ad}}= & A_{0} E^{*} \lambda\left(\frac{h_{0}}{\lambda}\right)^{2} 2(G(b, \alpha) \\
& \left.-\frac{b}{\pi \Theta} \int_{0}^{1} d x\left[1+\left(\frac{2 \pi h_{0}}{\lambda}\right)^{2} \sin ^{2}(2 b x)\right]^{1 / 2}\right),
\end{aligned}
$$

where $\Theta=E^{*} h_{0}^{2} / \Delta \gamma \lambda, b=\pi a / \lambda$, and 


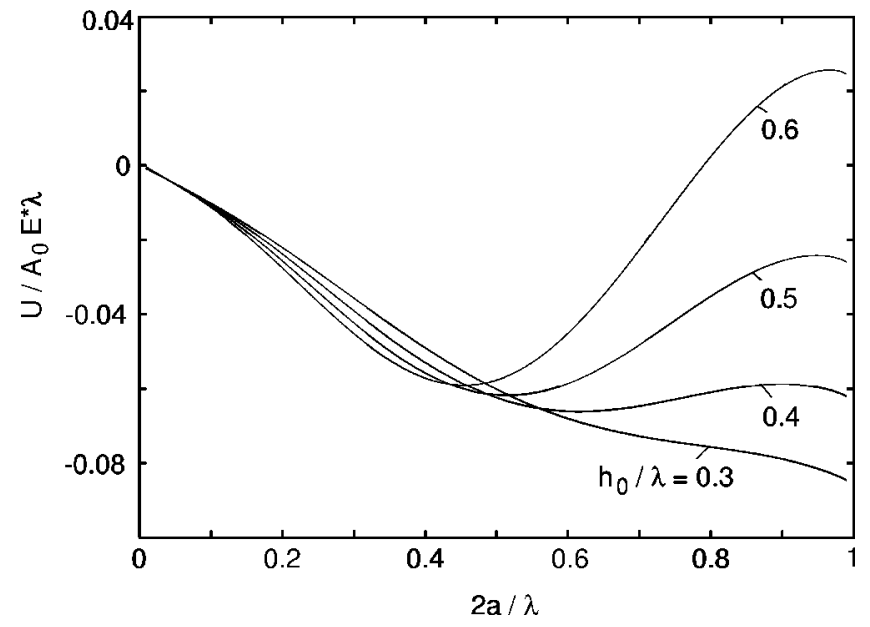

FIG. 2. The variation of total energy $U$ (in units of $A_{0} E^{*} \lambda$ ) with the relative contact area $2 a / \lambda$. Calculations are shown for four different surface corrugation amplitudes, $h_{0} / \lambda=0.3,0.4,0.5$, and 0.6. In the calculation we have used $E=500 \mathrm{MPa}$ and $\lambda=50 \AA$ and $\Delta \gamma=15 \mathrm{meV} / \mathrm{A}^{2}$.

$$
\begin{aligned}
G(b, \alpha)= & -\frac{\pi}{8} \sin ^{2} b\left(\sin ^{2} b-2\right) \\
& -b \alpha \int_{0}^{1} d x \cos (2 b x)\left[1-\left(\frac{\cos b}{\cos (b x)}\right)^{2}\right]^{-1 / 2} \\
& +\frac{b}{3}\left(1-\frac{2 \alpha}{\sin ^{2} b}\right) \sin ^{4} b\left[1-\left(\frac{\pi}{2 b}\right)^{2}\right]^{3 / 2} \\
& +b\left(1-\frac{2 \alpha}{\sin ^{2} b}\right)^{2} \sin ^{4} b\left\{( \frac { \pi } { 2 b } ) \operatorname { l n } \left[\left(\frac{\pi}{2 b}\right)\right.\right. \\
& \left.+\sqrt{\left(\frac{\pi}{2 b}\right)^{2}-1}\right]-\sqrt{\left.\left(\frac{\pi}{2 b}\right)^{2}-1\right\}}
\end{aligned}
$$

where

$$
\xi=\frac{\sin (b x)}{\sin b}, \quad \alpha=\frac{1}{2} \sin ^{2} b-\frac{\sigma \lambda}{2 \pi E^{*} h_{0}} .
$$

Note that for $\sigma=0$ we get $\alpha=(1 / 2) \sin ^{2} b$, which makes the prefactors zero in the third and fourth terms in Eq. (2), so that (for $\sigma=0$ )

$$
\begin{aligned}
G(b, \alpha)= & -\frac{\pi}{8} \sin ^{2} b\left(\sin ^{2} b-2\right)-b \alpha \int_{0}^{1} d x \cos (2 b x) \\
& \times\left[1-\left(\frac{\cos b}{\cos (b x)}\right)^{2}\right]^{-1 / 2} .
\end{aligned}
$$

Figure 2 shows the variation of total energy $U$ (in units of $A_{0} E^{*} \lambda$ ) with the relative contact area $2 a / \lambda$. Calculations are shown for four different surface corrugation amplitudes, $h_{0} / \lambda=0.3,0.4,0.5$, and 0.6 . In the calculation we have used $E=500 \mathrm{MPa}, \lambda=50 \AA$, and $\Delta \gamma=15 \mathrm{meV} / \AA^{2}$. Note that for $h_{0} / \lambda=0.4,0.5$, and 0.6 there are two local minima of $U$, one for complete contact $(2 a=\lambda)$ and another for partial contact $(2 a<\lambda)$.

The stable-state (at zero temperature) relative contact area $2 a / \lambda$ is determined by the global minima of $U$ as a
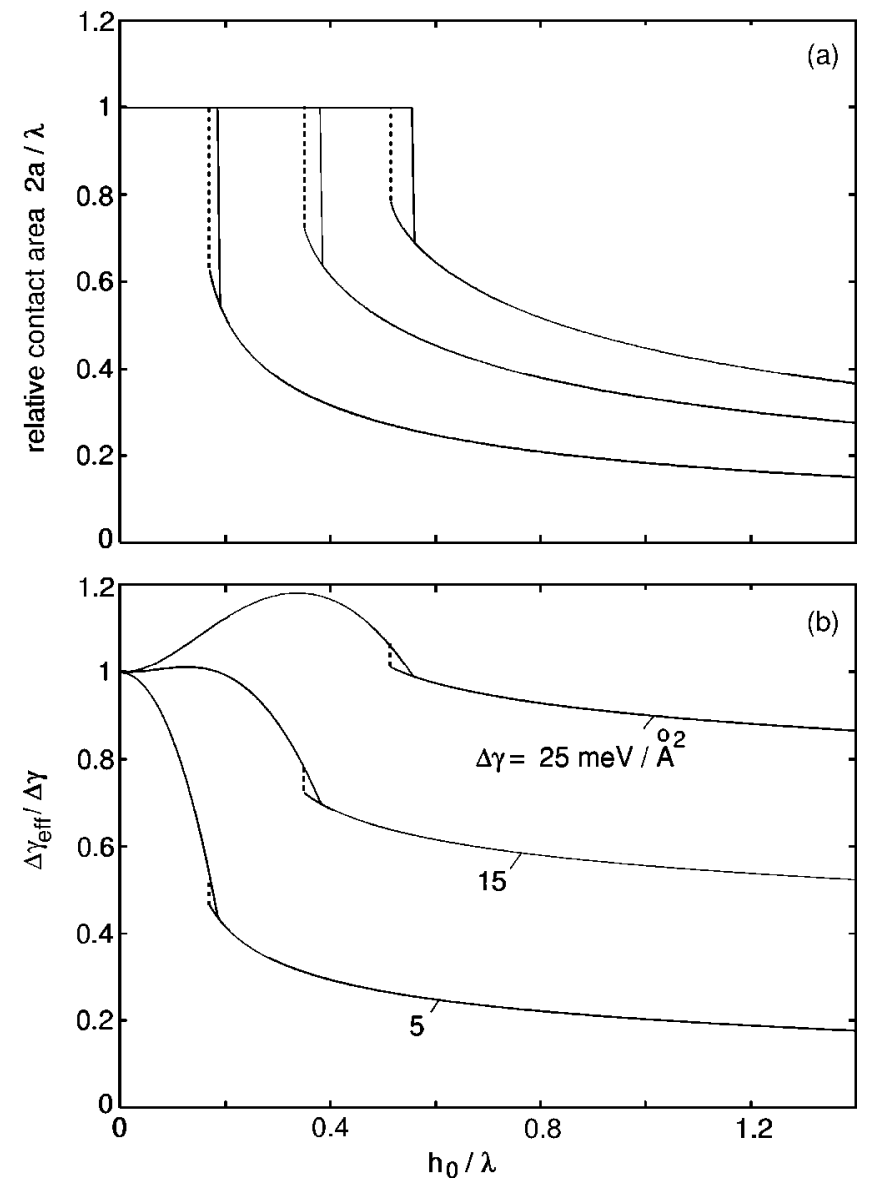

FIG. 3. The variation of (a) the relative contact area $2 a / \lambda$ and (b) the effective surface energy $\Delta \gamma_{\text {eff }}$ with the relative amplitude $h_{0} / \lambda$ of the surface corrugation. In the calculation we have used $E=500 \mathrm{MPa}$ and $\lambda=50 \AA$ and results are shown for $\Delta \gamma=25,15$, and $5 \mathrm{meV} / \AA^{2}$. The curves have been obtained by minimizing the total energy $U$ with respect to $a / \lambda$. The solid curves correspond to the total minimum free energy configuration, while the dashed curves show the behavior when starting in the partial detached state for large $h_{0}$ and then reducing $h_{0}$; in this case the system is trapped in a metastable state [local minima of $U(a)$, see Fig. 2] before finally flipping into the complete contact state at small enough $h_{0}$ (vertical dashed lines in the figures), when the local minima vanishes.

function of $a / \lambda$. Figure 2 shows that in general one may expect two local minima of $U$ as a function of $2 a / \lambda$, one corresponding to complete contact and another for partial contact. From Eq. (1) it is clear that $a / \lambda$ and $U / A_{0} E^{*} \lambda$ at a local minima of $U(a / \lambda)$ are functions only of the dimension less parameters $h_{0} / \lambda$ and $\Theta$. It is convenient to write

$$
U_{\text {min }}=-\Delta \gamma_{\text {eff }} A_{0},
$$

where

$$
\begin{aligned}
\Delta \gamma_{\mathrm{eff}}= & \Delta \gamma \times \min \left\{\frac{2 b}{\pi} \int_{0}^{1} d x\left[1+\left(\frac{2 \pi h_{0}}{\lambda}\right)^{2} \sin ^{2}(2 b x)\right]^{1 / 2}\right. \\
& -2 \Theta G(b, \alpha)\} .
\end{aligned}
$$

In the definition of $\Delta \gamma_{\text {eff }}$ we refer to the global minima. Figure 3 shows the variation of (a) the relative contact area $2 a / \lambda$ and (b) the effective surface energy $\Delta \gamma_{\text {eff }}$ with the amplitude $h_{0}$ of the surface corrugation, as obtained from 
curves such as those in Fig. 2. Results are shown for $\Delta \gamma$ $=25,15$, and $5 \mathrm{meV} / \AA^{2}$. The solid curves have been obtained by minimizing the total energy $U$ with respect to $a / \lambda$, while the dashed curves show the behavior when starting in the partial detached state for large $h_{0}$ and then reducing $h_{0}$; in this case the system is initially trapped in the partial detached state [local minima of $U(a / \lambda)$, see Fig. 2] before finally flipping into the complete contact state at small enough $h_{0}$ (vertical dashed lines in the figures), when the local minima vanishes. Note that, in accordance with the discussion in the beginning of this section, the transition from the partial detached state to complete contact occurs when $\Theta \approx 1$ or $h_{0} / \lambda \approx(\Delta \gamma / E \lambda)^{1 / 2}$. The solid line in Fig. 3 is composed of two branches with discontinues derivative at the intersection point. The left branch corresponds to the fully attached state, and the right branch to a partial contact state.

Note that within the elastic continuum model (for an infinite system), with infinitesimal short-ranged wall-wall interaction (so called, contact interaction) and at zero temperature, the complete contact state is stable for arbitrary high applied pull-off stress. The reason originates from the infinitesimal extent of the wall-wall interaction potential: an infinite large stress must be applied in order to break the block-substrate bond [in such a way that the product of the (infinite) stress and the (vanishing) bond distance equals the (finite) surface energy $\Delta \gamma$ ]. At the complete contact state (for an infinite system) there are no crack edges (where the stress would diverge) so that any finite applied stress will give rise to a finite stress everywhere at the interface. Thus, within the present model the complete contact state is stable for any applied external stress. In reality, with a finite extent of the wall-wall interaction potential (as is the case in the molecular dynamics simulations presented in Sec. III), the complete contact will break at finite pull-off stress. At finite temperature the breaking of the full contact will usually occur by the thermal nucleation of a penny-like detached area (crack) in the highest tensile stress regions of the blocksubstrate interface. In the present case this is at the bottom of a valley in the substrate roughness profile. For a finite system the bond-breaking (detachment) may occur by crack propagation, from the periphery of the contact area toward the center. However, exactly how the nucleation and propagation of the crack occur does not interest us here.

The effective surface energy $\Delta \gamma_{\text {eff }}$ can be used directly to determine the pull off force. For example, the pull off force of an elastic spherical body (e.g., a rubber ball) from a hard substrate is given by the standard formula ${ }^{10} F_{N}$ $=(3 \pi / 2) R \Delta \gamma_{\mathrm{eff}}$. This formula is strictly valid only for elastically soft solids, and for rough surfaces only if the ball radius $R$ is large enough (compared to $h$ ) as discussed in Ref. 15 .

\section{COMPUTER SIMULATIONS}

The above-presented theory is valid for any length scale where continuum mechanics is applicable, and it is interesting to assess to what extent these predictions hold when system size is decreased to nanoscale. We have performed an extensive series of molecular dynamics (MD) simulations for the same surface roughness profile as used previously, and for more complex roughness profiles, with and without thin liquid contamination films. These results will be reported elsewhere, ${ }^{15}$ and here we only give a few results related to the discussion above.

We have used the methodology described in Ref. 16 for constructing the solid walls, with effective springs chosen such that long range elastic properties are reproduced. The walls were composed of single layer of atoms in a simple cubic geometry, with stiff springs connecting neighboring atoms, and soft springs connecting the atoms to a hard wall; the spring constants were chosen so as to reproduce both the short- and the long-range elastic properties of the solids. In all simulations we were using a hard substrate (elastic modulus $E=77 \mathrm{GPa})$ and an elastically "soft" $(E=0.5 \mathrm{GPa})$ block, of vertical width 0.2 and $100 \AA$, respectively. Block and substrate atoms interacted via pairwise additive LennardJones potential chosen so that the change in the surface energy $\Delta \gamma \approx 15 \mathrm{meV} / \AA^{2}$ at $T=0 \mathrm{~K}$.

It should be stressed that the analytical model and the computer simulation model involve very different approximations of the same physical phenomena. In the analytical model the adhesion interaction is infinitely short ranged, and the surfaces are assumed to be able to slide relative to each other without friction so that the tangential stress at the interface vanishes. We have also assumed that $\Delta \gamma$ does not depend on the surface area, which should be a good approximation for materials such as rubber or gelatin, which have high-mobility (liquid-like) surface layers. The MD simulations, on the other hand, contain a discrete (and constant) number of atoms, the adhesion interaction has a finite extent and the elastic properties are treated in an approximate manner. Moreover, as we vary the height of the corrugation (at a fixed number of particles) we change the local particles density and hence the local surface energy $\Delta \gamma$, in contrast to the analytical model where $\Delta \gamma$ is constant. Despite the differences, the two approaches agree qualitatively very well and quantitatively to within a factor of $2-3$, as demonstrated in the following.

We consider first a cosine corrugation in the $x$ direction and constant in the $y$ direction; this case can be directly compared to the theoretical predictions presented in Sec. II. The substrate atoms were connected to a rigid surface of the form:

$$
h(x, y)=h_{0} \cos \left(\frac{2 \pi x}{\lambda}\right) .
$$

Figure 4 shows simulation snapshots (side view) during decrease and increase of the surface roughness amplitude.

In the simulation discussed in the following we used a cell of dimension $L_{x}=\lambda=100 \AA$ and $L_{y}=100 \AA$, in the $x$ and $y$ directions, respectively. The substrate consist of $39 \times 39$ atoms and the block of $37 \times 37$ atoms. The cell extend from $-L / 2$ to $L / 2$ along both the $x$ and $y$ axes.

At $T=0 \mathrm{~K}$, as shown in Fig. 5(a), the transitions between full contact and partial contact are abrupt. The transitions full $\rightarrow$ partial contact and the opposite partial $\rightarrow$ full contact occur at different roughness heights, i.e., hysteresis is observed during roughness variation. It is evident that over 

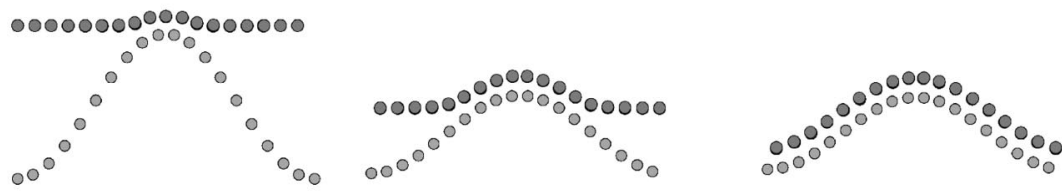

(a)

Decreasing corrugation height

(b)

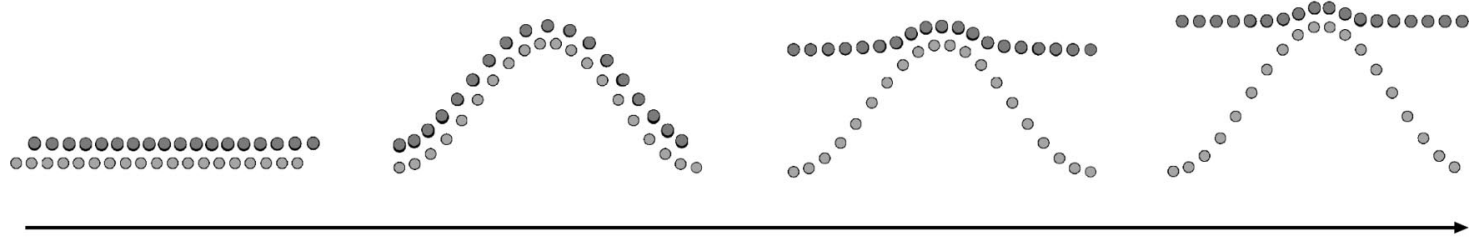

Increasing corrugation height

FIG. 4. A series of simulation snapshots (side view) illustrating roughness variation in the cylindrical symmetry case. Here we had a small system of $50 \times 50 \AA$, and surface energy $\Delta \gamma \approx 1 \mathrm{meV} / \AA^{2}$. (a) Reducing roughness height. The two middle snapshots shows the state of the system just before and right after snapping to full contact. (b) Increasing roughness height. The two middle snapshots shows the state of the system just before and right after snapping between full contact and partial detachment.

the range of roughness heights $h_{0} / \lambda \sim 0.15-0.8$ there exists two stable energy minima. Figure $5(\mathrm{~b})$ shows the results for $\Delta \gamma_{\text {eff }}$ as a function of $h_{0} / \lambda$. It shows that for $h_{0} / \lambda<0.3$ the full contact state is the global minima, and for higher values the partial contact is the global minima. Given the different methodologies and assumptions, all simulations results are in very good qualitative and quantitative agreement with the predictions of the analytical theory (compare with Fig. 3).

Figures 5(c) and 5(d) show the results ${ }^{17}$ of the same roughness variations as before, but at $T=30 \mathrm{~K}$. When comparing to $T=0 \mathrm{~K}$ [Figs. 5(a) and 5(b)] one can clearly see that at $T=30 \mathrm{~K}$ the transition full $\rightarrow$ partial contact is significantly shifted (from $h_{0} / \lambda \approx 0.8$ to 0.6 ) toward lower corrugation height, whereas the transition partial $\rightarrow$ full contact is
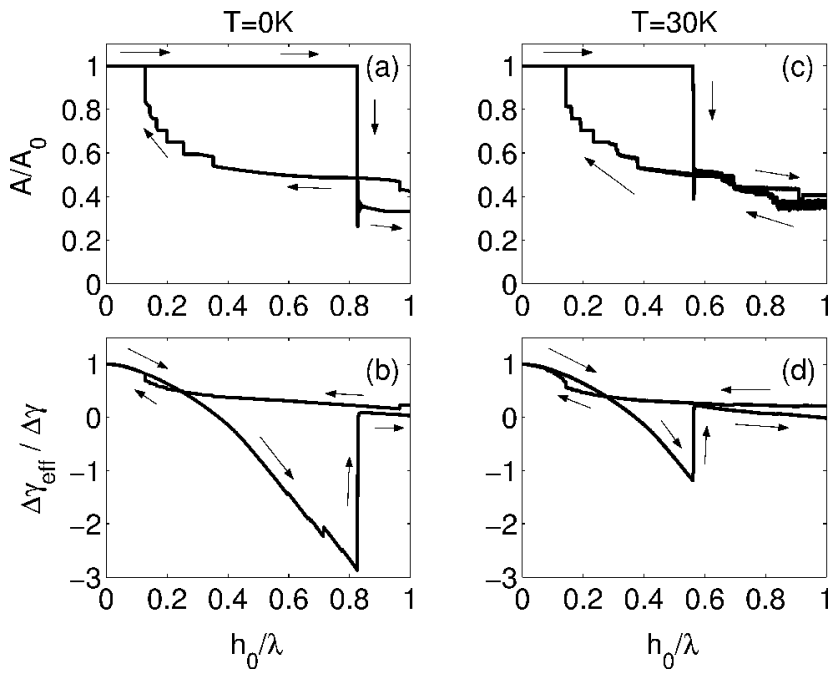

FIG. 5. Simulation results for roughness ramping in the cylindrical symmetry case, at $T=0$ and $30 \mathrm{~K}$. The $x$ axis is the corrugation height normalized by the wavelength $\lambda=L_{x}$. (a) and (c): contact area at $T=0 \mathrm{~K}$ and $T=30 \mathrm{~K}$. (b) and (d): Effective surface energy (Ref. 17) at $T=0 \mathrm{~K}$ and $T=30 \mathrm{~K}$, normalized by the surface energy for flat surfaces in full contact (at each temperature). The arrows are used to guide the eyes in the direction of height variation. much less affected, and actually shifted up from $h_{0} / \lambda$ $\approx 0.13$ to 0.14 . These observations are the results of both thermodynamic and kinetic effects. The thermodynamic effects comes from the entropic contribution (as discussed in Sec. IV) to the free energy, which affects the two contact states differently. Kinetic effects are briefly discussed in the following.

In Sec. II we pointed out that even if the partial contact state has the lowest free energy, in the continuum model (with a contact interaction between the walls), the complete contact state will be stable at zero temperature, independent of the magnitude of external applied stress. When the wallwall interaction potential has a finite extent, as in the present case, the complete contact state (at $T=0 \mathrm{~K}$ ) will only be stable as long as the tensile stress at the interface is everywhere below the critical value $\sigma^{*}$ necessary to break the wall-wall adhesive bonding. Thus, in the present case at $T=0 \mathrm{~K}$, the block substrate bond will break when the tensile stress at the bottom of a valley reaches $\sigma^{*}$. However, at finite temperatures the transition from complete to partial contact will occur by the thermal nucleation of a pennyshaped detached area (crack). This will occur even if the stress at the bottom of a well is below $\sigma^{*}$. This is, at least in part, the reason why at $T=30 \mathrm{~K}$ the transition from the full to the partial contact state is significantly shifted toward lower corrugation height. Note also that when the system is in the partial contact state there is already a crack at the interface, and there is no reason to expect the transition from the partial contact to the full contact to depend strongly on the temperature, as observed.

We have also considered a system with a Gaussian corrugation

$$
h(x, y)=h_{0} \exp \left(\frac{x^{2}+y^{2}}{2 s^{2}}\right),
$$

where $s=0.2 L_{x}$ is the width of the Gaussian. Note that $h_{0}$ here represents the height of the asperity, while in the cosine asperity (discussed previously) $h_{0}$ was the amplitude (half 

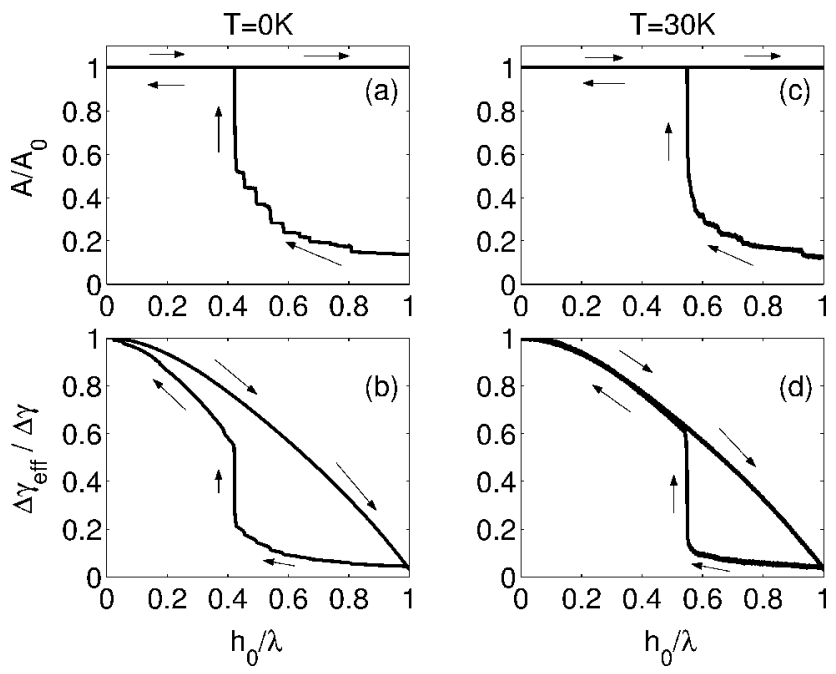

FIG. 6. Simulation results for roughness variation of a Gaussian corrugation, at $T=0$ and $30 \mathrm{~K}$. The $x$ axis is the corrugation height normalized by the wavelength $\lambda=L_{x}$. (a) and (c): Contact area at $T=0 \mathrm{~K}$ and $T=30 \mathrm{~K}$. (b) and (d): Effective surface energy (Ref. 17) at $T=0 \mathrm{~K}$ and $T=30 \mathrm{~K}$, normalized by the surface energy for flat surfaces in full contact (at each temperature). The arrows are used to guide the eyes in the direction of height variation.

the height). Figure 6 shows the results of roughness variation for this geometry. Qualitatively the cylinder (Fig. 5) and Gaussian (Fig. 6) cases yield similar results, but there are some important quantitative differences: First, it is obvious that for the Gaussian asperity the contact area in the partial contact state is very small. This is easy to understand since the surfaces make contact only at the asperity top, and not along a line as for the cylinder asperity case. Second, due to the high value of the surface energy $\Delta \gamma$, we could not obtain in the simulations a detachment transition for this geometry. Note also that the point at which the partial $\rightarrow$ full contact occur [Figs. 6(a) and 6(c)] is shifted to higher $h_{0} / \lambda$ as the temperature is raised, and the temperature sensitivity is somewhat higher than in the cylinder case. Figure 6(b) shows that the surface energy after the partial $\rightarrow$ full contact transition does not overlap with the value obtained from the simulations where roughness height is increased. The reason is that after the partial $\rightarrow$ full contact transition, the system was trapped in a local (nonglobal) minima with parallel stresses which could not be relieved by thermal motion since $T=0 \mathrm{~K}$. At higher temperature in contrast [Fig. 6(d)], we get a very good overlap.

\section{FINITE TEMPERATURES: ROLE OF THE VIBRATIONAL ENTROPY}

The derivations in Sec. II were for zero temperature. Temperature may influence the adhesion in many ways, e.g., the elastic constants and the contact energy $\Delta \gamma$ will depend on the temperature, but these effects are easy to take into account (if the temperature dependence is known, e.g., from experiments) and does not invalidate the theory presented in Sec. II. On the other hand there are additional thermal effects which are not included, and have to be considered separately. Kinetic effects, such as thermally activated processes (e.g., thermal nucleation of cracks as discussed in Sec. III) may be very important, and are not included in the treatment presented in Sec. II. Here we present a simple discussion about another important thermodynamic temperature effect, namely the role of the vibrational entropy. We will study the free energy $\mathcal{F}$, and we show that the contribution to $\mathcal{F}$ from the vibrational entropy favors the detached state.

We have shown in Sec. II that at zero temperature, for a given range of corrugation heights, there are two minima in the total energy versus contact area. If the amplitude $h_{0}$ of the surface roughness is small, the global minima correspond to a fully attached state. If the elastic solid binds strongly to the hard substrate, the combined system will have relatively high-frequency interfacial vibrational modes and we can neglect their contribution to the vibrational entropy. However, the free surface of the elastic solid will have low frequency phonon modes which will give a large contribution to the vibrational entropy of the unbound state.

The thermodynamic free energy is $\mathcal{F}=E-T S$ where $E$ is the internal energy [given by Eq. (1) at zero temperature], $T$ is the temperature and $S$ is the entropy. At zero temperature the free energy is identical to the internal energy. At finite temperature the entropy is important, since the partially detached state the system has much greater vibrational entropy compared to the full contact state, thus lowering the free energy of the detached state compared to its original value at $T=0 \mathrm{~K}$. In our model we assume that the surface vibrational entropy of the elastic solid is completely suppressed at full contact because of the rigid nature of the substrate. It is easy to estimate the change in the vibrational entropy as we change the contact area. The contribution to the free energy from elastic surface waves can be expressed as a sum over the relevant normal modes. The free energy of a set of phonons is ${ }^{18}$

$$
\begin{aligned}
\mathcal{F} & =\mathcal{F}_{0}+k_{B} T \sum_{n} \log \left[1-\exp \left(-\frac{\hbar \omega_{n}}{k_{B} T}\right)\right] \\
& =\mathcal{F}_{0}+k_{B} T \int_{0}^{\omega_{\max }} d \omega \rho(\omega) \log \left[1-\exp \left(-\frac{\hbar \omega}{k_{B} T}\right)\right],
\end{aligned}
$$

where the summation goes over all normal modes, $\omega_{n}$ is the frequency of the $n$th normal mode, $k_{B}$ is the Boltzmann constant, and $\rho(\omega)$ is the phonon density of states. The cut-off frequency $\omega_{\max }$ is the maximal surface phonon frequency. In the elastic continuum model, the elastic displacement of the surface of a semi-infinite solid is the result of the Rayleigh phonons and longitudinal and transverse bulk phonons. However, here we take the simplest possible approach and just include the Rayleigh phonons. The contact area between the surfaces is in the form of a long strip. The Rayleigh phonons can propagate only on the free (or unbound) surface of the elastic solid with dimensions $L_{x}=\lambda-2 a$ and $L_{y} \gg L_{x}$ [we assume that the edges $x= \pm a \pm n \lambda(n=1,2, \ldots)$ are rigidly attached to the substrate]. We simplify things further by neglecting the small wave vector cut off which results from the finite width $L_{x}$. These are obviously crude approximations but they serve to explain some qualitative aspects of quite general validity. Therefore it is easy to show that the density of states is 


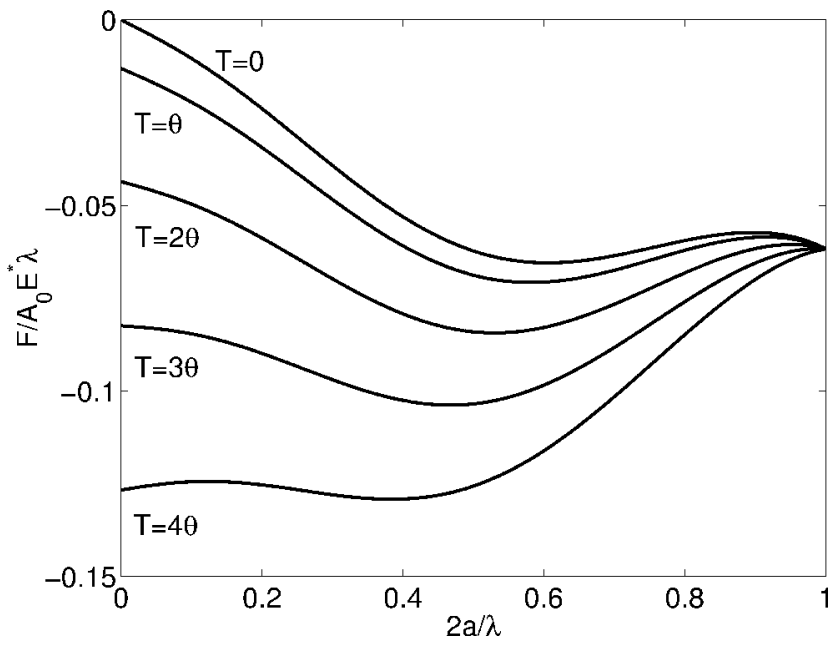

FIG. 7. Variation of free energy $\mathcal{F}$ as a function of contact area, for various temperatures and fixed corrugation height $h_{0} / \lambda=0.4$.

$$
\rho(\omega) d \omega=\frac{L_{x} L_{y}}{2 \pi c_{\mathrm{R}}^{2}} \omega d \omega,
$$

where $c_{R}$ is the velocity of the surface waves. We can calculate $\omega_{\max }$ from the normalization requirement [using Eq. (6)]

$$
\begin{aligned}
& \int_{0}^{\omega_{\max }} \rho(\omega) d \omega=N, \\
& \omega_{\max }^{2}=\frac{4 \pi N c_{R}^{2}}{L_{x} L_{y}}=\frac{4 \pi c_{R}^{2}}{l_{b}^{2}},
\end{aligned}
$$

where $N=N_{x} N_{y}$ is the number of atoms and $l_{b}$ is the typical bond length. Combining Eqs. (5) and (6), and setting $x$ $=\hbar \omega / k_{B} T$ yields

$$
\mathcal{F}=\mathcal{F}_{0}+\frac{L_{x} L_{y}}{\pi c_{R}^{2}} \frac{\left(k_{B} T\right)^{3}}{\hbar^{2}} \int_{0}^{\theta / T} x \log \left(1-e^{-x}\right) d x .
$$

Here $\theta$ is the characteristic temperature

$$
\theta=\frac{\hbar \omega_{\max }}{k_{B}} .
$$

If the width of the contact area is $2 a$, it is easy to see from Fig. 1 that $L_{x}=\lambda-2 a$, where $\lambda$ is the corrugation wavelength. Therefore the expression for the free energy becomes

$\mathcal{F}=\mathcal{F}_{0}+\frac{L_{y}}{\pi c_{R}^{2}} \frac{\left(k_{B} T\right)^{3}}{\hbar^{2}}(\lambda-2 a) \int_{0}^{\theta / T} x \log \left(1-e^{-x}\right) d x$.

Note that the result of the integral in Eq. (8) is always negative. Therefore Eq. (8) shows that for a given temperature $T$, the zero temperature free energy [Eq. (1)] is supplemented by a term that increases the total free energy as the contact area is increased. Thus, for $T>0 \mathrm{~K}$ the relative magnitude of the two minima as well as the above-discussed barrier change, lowering the free energy of the partially detached state and thus stabilizing it.

Figure 7 shows the variation of free energy $\mathcal{F}$ as a function of contact area, for various temperatures and fixed corrugation height $h_{0} / \lambda=0.4$. In calculating those curves we
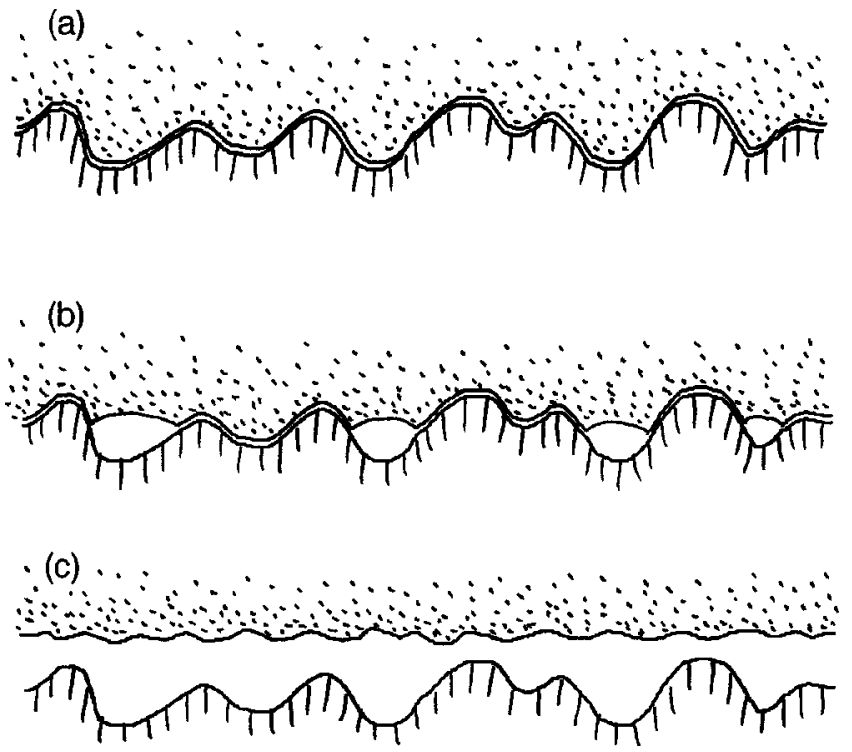

FIG. 8. The contact between an elastic solid (dotted area) and a hard rough substrate. (a) At low temperature complete contact occur between the solids. (b) As the temperature increases local detachments occur at the interface. This leads to an increase in the vibrational entropy which is the driving force for the detachments. (c) At high temperature a complete detachment transition occurs.

have used the same parameters as before, assuming bond length $l_{b}=2.7 \AA$ and the sound velocity $c_{R}=730 \mathrm{~m} / \mathrm{s}$ (chosen in accordance with simulation parameters). As expected, increasing the temperature stabilizes the partially detached state and thus makes it the favorable one. Moreover, the barrier for complete detachment also keeps decreasing as we increase the temperature, and at high enough temperatures it disappears completely, making the stable state correspond to complete detachment (no contact at all).

The stabilization of the detached state with increasing temperature is consistent with our MD observations: as we increase temperature the transitions between the full and partial contact states occur at smaller roughness amplitude. As discussed in Sec. III, the strong reduction observed for the (complete contact $\rightarrow$ partial contact) transition may be due mainly to the thermal nucleation of a crack, but there will also be a contribution from the vibrational entropy effect discussed here.

Finally, we note that the influence of temperature on adhesion has been observed for biological membranes. At "low" temperature a membrane may be (weakly) bound to a (hard) solid wall, or many membranes may be bound together in a stack. In these cases, when the temperature increases a debounding transition to free membranes is often observed. ${ }^{19-21}$ This is again due to the loss of vibrational (or conformational) entropy as the membrane is confined in the adhesive state. Thus, thermal shape fluctuations renormalize the direct wall-wall interaction, increasing its repulsive part. The renormalized interaction may be attractive or repulsive at large membrane separation, corresponding to a bound or an unbound state of the membranes. These two states are separated by a phase boundary at which the membranes undergo an unbinding or adhesion transition. What we have shown above is that in many cases there may be a partial 
detachment transition before complete detachment occurs, see Fig. 8. This latter scenario is, e.g., expected when a membrane (or an elastic solid) bind to a hard substrate with surface roughness. For random surface roughness on many different length scales, thermally induced detachments are likely to increase continuously as the temperature is increased toward a critical temperature $T_{c}$, and for $T>T_{c}$ the walls are completely separated. The corresponding phase transition is likely to be continuous. We suggest that it may be possible to study the unbounding transition for rubber in contact with a rough glass substrate if the right elastic modulus and surface energy is chosen for the rubber. However, since the elastic modulus $E$ of rubber increases with increasing temperature (which would tend to induce detachments), it is necessary to know the temperature dependence of $E(T)$ accurately, in order to determine the exact origin of the detachments.

\section{SUMMARY}

We have presented an exact solution to a simple model of adhesion between an elastic body and a hard substrate with periodic roughness. When the two surfaces are in contact at temperature $T=0 \mathrm{~K}$ they can adhere in either full contact (following exactly the roughness topography) or in partial contact, where contact only occurs close to the tops of the asperities. The preferred state is determined by a dimensionless variable $\Theta$ which is the ratio between the elastic energy and the adhesion energy. For $\Theta \gg 1$ the system will be in partial contact state, while for $\Theta \ll 1$ full contact occur.

In the intermediate range $\Theta \sim 1$ the system may be in either state, depending on the initial conditions. In these cases, as a function of the contact area, we may have two energy minima, with some potential barrier separating them. At nonzero temperature thermally activated transitions can occur between the two states. We have shown how temperature affects the contact mechanics. In particular, increasing the temperature stabilizes the partial contact state (because of the increase of the vibrational entropy), and at even higher temperatures there is a complete detachment transition.

Molecular dynamics simulations were employed to study nanoadhesion. We considered two idealized roughness geometries. The simulations are in good qualitative agreement with the analytical calculations, and even quantitatively to within a factor of $2-3$.

\section{ACKNOWLEDGMENTS}

The authors thank A. Nitzan, K. L. Johnson, and P. Ballone for useful discussions. The authors also thank BMBF for a grant related to the German-Israeli Project Cooperation "Novel Tribological Strategies from the Nano-to Meso-Scales." B.N.J.P. thanks the EC for a "Smart QuasiCrystals" grant under the EC Program "Promoting Competitive and Sustainable growth." S.Z. acknowledges the hospitality of the research center in Jülich Germany and its staff while doing part of this work.

${ }^{1}$ K. L. Johnson, Contact Mechanics (Cambridge University Press, Cambridge, 1987); B. N. J. Persson, Sliding Friction: Physical Principles and Applications, 2nd ed. (Springer, Heidelberg, 2000); J. Israelachvili, Intermolecular and Surface Forces, 2nd ed. (Academic, London, 1995).

${ }^{2} \mathrm{~K}$. Kendall, Molecular Adhesion and Its Applications: The Sticky Universe (Plenum, New York, 2001).

${ }^{3}$ D. Maugis, Contact, Adhesion and Rupture of Elastic Solids (Springer, Berlin, 2000).

${ }^{4}$ C. Gay and L. Leibler, Phys. Today 1999, 48.

${ }^{5}$ B. N. J. Persson, J. Chem. Phys. 115, 3840 (2001); Phys. Rev. Lett. 87, 116101 (2001).

${ }^{6}$ B. N. J. Persson and E. Tosatti, J. Chem. Phys. 115, 5597 (2001).

${ }^{7}$ B. N. J. Persson, Eur. Phys. J. E 8, 385 (2002).

${ }^{8}$ S. Zilberman and B. N. J. Persson, Solid State Commun. 123, 173 (2002); 124, 227 (2002).

${ }^{9}$ K. N. G. Fuller and D. Tabor, Proc. R. Soc. London, Ser. A 345, 327 (1975).

${ }^{10}$ K. L. Johnson, K. Kendall, and A. D. Roberts, Proc. R. Soc. London, Ser. A 324, 301 (1971).

${ }^{11}$ C. Y. Hui, Y. Y. Lin, and C. Creton, J. Polym. Sci., Part B: Polym. Lett. 40, 545 (2002).

${ }^{12}$ K. L. Johnson, Int. J. Solid Struct. 32, 423 (1995).

${ }^{13}$ H. M. Westergaard, Trans. ASME, J. Appl. Mech. 6, 49 (1939).

${ }^{14}$ W. T. Koiter, Ing. Arch. 28, 165 (1959).

${ }^{15} \mathrm{~S}$. Zilberman and B. N. J. Persson (in preparation).

${ }^{16}$ B. N. J. Persson and P. Ballone, J. Chem. Phys. 112, 9524 (2000).

${ }^{17}$ The surface energy $\Delta \gamma_{\text {eff }}$ in this case is defined in terms of differences in internal energy and not free energy. Nevertheless, the qualitative behavior is similar to our expections from the free difference based on the analytical model.

${ }^{18}$ L. D. Landau and E. M. Lifshitz, Statistical Physics, 3rd ed., Part 1 (Pergamon, New York, 1980).

${ }^{19}$ R. M. Servuss and W. Helfrich, J. Phys. (France) 50, 809 (1989).

${ }^{20}$ U. Seifert and R. Lipowsky, Phys. Rev. A 42, 4768 (1990).

${ }^{21}$ R. Lipowsky, Nature (London) 349, 475 (1991). 